\title{
Uma nova ferramenta de fórum de discussão para ambientes virtuais de aprendizagem Moodle: o plug-in Octopus
}

Priscilla B. Mendes ${ }^{1}$, Josiane L. Machiavelli ${ }^{1,4}$, Rodrigo C. Lins ${ }^{1,3}$, Patrícia C. de A. R. Tedesco ${ }^{1,3}$, Cristine M. G. de Gusmão ${ }^{1,2}$

${ }^{1}$ Grupo de Pesquisa SABER Tecnologias Educacionais e Sociais - Universidade Federal de Pernambuco (UFPE) - Recife - PE - Brasil

${ }^{2}$ Departamento de Engenharia Biomédica - Universidade Federal de Pernambuco (UFPE) - Recife - PE - Brasil

${ }^{3}$ Centro de Informática, Universidade Federal de Pernambuco (UFPE) - Recife - PE Brasil

${ }^{4}$ Pós-graduação em Educação Matemática e Tecnológica - Universidade Federal de Pernambuco (UFPE) - Recife - PE - Brasil

\{priscy285, josiane.machiavelli, rodrigolinsbr, cristinegusmao\}@gmail.com, pcart@cin.ufpe.br

\begin{abstract}
Providing educational resources that are able to effectively promote students' participation in a context of technology-based education, where users' interactions are characterized by occurring in different times and physical spaces, is a challenge that requires innovative solutions. The present work describes the ideation and creation of a new forum tool, a plug-in for the Moodle platform named Octopus, that has several enhancements in comparison with traditional online forums. Its most important characteristics are a recommendation mechanism based on Artificial Intelligence and the generation of detailed reports of the forum's general activities.
\end{abstract}

Resumo. Fornecer recursos educacionais que promovam a participação efetiva dos estudantes num contexto de educação mediada por tecnologia, em que a interatividade entre os usuários caracteriza-se por ocorrer em espaços físicos e tempos diferenciados, é um desafio que requer soluções inovadoras. $O$ trabalho aqui apresentado descreve a concepção e criação de uma nova ferramenta para ambientes virtuais de aprendizagem, um plug-in de fórum para o Moodle denominado Octopus, que possui diversos aprimoramentos com relação aos fóruns on-line tradicionais, como mecanismo de recomendação baseado em inteligência artificial, e a geração de relatórios que exibem dados detalhados a respeito das atividades gerais do fórum.

\section{Introdução}

O diagnóstico de uma necessidade de maior interação e engajamento nos fóruns motivou a criação de um novo plug-in, pensado para suprir aspectos não atendidos pela atividade tradicional de fórum da plataforma Moodle, e ainda permitir novas possibilidades pedagógicas. 
Os maiores diferenciais desta ferramenta são a utilização de tecnologia de inteligência artificial para realizar recomendações personalizadas de postagens e materiais aos usuários, o que permite maior efetividade no estímulo à participação, além de sugerir conteúdos condizentes com os interesses e necessidades dos participantes, e possibilitar a geração de relatórios que apresentam uma visão detalhada da participação dos usuários no Octopus.

\section{Cenário de Uso}

A ferramenta descrita no presente estudo foi desenvolvida pelo SABER Tecnologias Educacionais e Sociais, um grupo de pesquisa ligado à Universidade Federal de Pernambuco, que conta com uma equipe multidisciplinar para desenvolver projetos nas áreas social e de educação.

O contexto específico no qual o plug-in foi utilizado, em princípio, foi nos cursos autoinstrucionais do Programa de Qualificação para Profissionais de Regulação do Sistema Único de Saúde, uma iniciativa fomentada pelo Ministério da Saúde por meio da Universidade Aberta do Sistema Único de Saúde. Tais cursos possuem um viés bastante prático na utilização dos conteúdos, considerando que visam formar profissionais para a atuação no contexto da Regulação em Saúde, sendo assim, o plug-in promoveu um importante papel ao possibilitar a formação de uma rede para que estes profissionais compartilhem suas experiências e pontos de vista sobre os assuntos abordados.

No entanto, a ferramenta tem potencial para ser utilizada em qualquer contexto onde haja utilidade para discussões on-line, onde os participantes podem promover a troca de ideias e o compartilhamento de conteúdos, seja esta a atividade principal, ou de forma complementar a outros recursos empregados na construção do conhecimento. Como exemplo, podemos citar ambientes de educação a distância puramente virtuais ou de blended learning e plataformas para educação corporativa nas empresas.

\section{Justificativa Pedagógica}

Os fóruns de discussão são recursos importantes em atividades realizadas em ambientes virtuais de aprendizagem, visto que proporcionam interações e compartilhamento de opiniões [Premagowrie, Vaani \& Ho, 2014], favorecendo a aprendizagem colaborativa. Eles permitem que os estudantes postem mensagens em tópicos de discussão, interajam e recebam feedback de outros usuários, e promovem uma compreensão mais profunda dos conteúdos estudados [Balaji \& Chakrabarti, 2010]. Assim, eles podem funcionar como instrumentos tanto de fixação do conteúdo quanto de avaliação de desempenho, uma vez que a qualidade das ideias apresentadas nas postagens dos estudantes pode ser reveladora quanto ao nível de compreensão e aprofundamento que estes dispõem dos assuntos abordados. No entanto, apesar de tais discussões serem um elemento de grande importância no processo de aprendizado, é preciso que haja incentivo à participação [Andresen, 2009], pois o potencial didático desta ferramenta só é realizado se os estudantes de fato se engajarem a ela [Mason, 2011].

A criação do plug-in descrito neste trabalho foi motivada pela percepção de uma defasagem do fórum tradicional do Moodle, que mantém seu formato basicamente 
inalterado desde versões bem antigas, por sua vez assemelhando-se aos fóruns tradicionais em diversas plataformas, os quais possuem apenas funcionalidades básicas de postagens e comentários. A simplicidade de tais recursos dificultava o trabalho de tutores e moderadores para estimular a discussão, já que este era baseado apenas em conteúdo apresentado numa interface pouco inspiradora. Sentiu-se a necessidade, portanto, de incorporar conceitos típicos das redes sociais, como tags, curtidas e contatos, motivando uma maior participação dos usuários no fórum ao fazer uso da familiaridade que estes já possuem com tais elementos em um contexto informal. Além disso, foi empregado o conceito de gamificação, o qual envolve o uso de elementos de jogos, tais como pontuações, níveis e conquistas, em outros contextos, o que promove uma motivação externa e mudança de comportamentos (NICHOLSON, 2012). Isto tem utilidade no âmbito educacional, no sentido de que é uma forma de influenciar os estudantes para que eles tenham uma postura atuante e participativa na aquisição do conhecimento.

Outro aspecto de melhoria observado foi de capturar informações a respeito das atividades no fórum que possibilitasse uma avaliação mais robusta, tanto no nível individual do usuário, quanto em termos gerais da participação dos integrantes do curso.

\section{Desenvolvimento}

O fórum Octopus foi desenvolvido como um plug-in do Moodle, de acordo com as diretrizes especificadas pela comunidade de desenvolvimento da plataforma.

O ponto de partida do processo de desenvolvimento foi o estabelecimento dos requisitos iniciais, seguido de um brainstorming para a detecção de outras funcionalidades relevantes, envolvendo as equipes da Coordenação Técnico-pedagógica e Supervisão Acadêmica do Grupo SABER, que representam mais fielmente a visão dos usuários, visto que mantém contato direto com alunos, professores e tutores, e pessoas da equipe de tecnologia da informação, os responsáveis pelo desenvolvimento do novo plug-in. Com as informações levantadas, ficou clara a necessidade de um mecanismo de recomendação de postagens e materiais didáticos, o que potencializa o engajamento dos usuários ao notificá-los periodicamente de itens de seu interesse. Este tipo de funcionalidade, em geral, é baseado em tecnologia de inteligência artificial, que possibilita a adaptabilidade e a personalização do sistema, baseando-se em dados brutos, o que dispensa a necessidade de constantes configurações para mantê-lo atualizado.

Ao serem definidos todos os requisitos e os recursos humanos necessários, verificou-se junto à Coordenação Geral a viabilidade da execução do novo projeto, considerando os custos dos recursos, notadamente constituído pelo custo das horas de trabalho da equipe, já que o plug-in foi desenvolvido inteiramente em plataforma opensource, e a alocação das atividades deste projeto em meio a outros trabalhos desenvolvidos pela equipe. A partir daí, foi estabelecido o cronograma, que também levou em consideração o calendário dos cursos em que a ferramenta seria inicialmente experimentada.

Com base nos requisitos definidos, criou-se um wireframe, isto é, um rascunho da aparência básica esperada para a ferramenta, demonstrando as visões dos usuários com o perfil de estudante e moderador. Isto serviu para criar o projeto gráfico mais detalhado, contemplando as informações necessárias para a caracterização da peça gráfica. Assim, o plug-in teve sua implementação iniciada, utilizando-se o sistema de 
controle de versão GitLab para a escrita do código, permitindo que este processo se desse de maneira organizada e facilitando sua rastreabilidade.

Após o desenvolvimento do plug-in, iniciou-se um período de testes, cuja etapa inicial foi realizada por dois componentes da equipe de desenvolvimento. Ao serem corrigidos os erros iniciais detectados por estes, outros testes foram realizados, envolvendo uma equipe de perfil diversificado, que possuía uma visão não enviesada da ferramenta, visto que as pessoas que a compuseram não participaram do processo de elicitação de requisitos. Novas correções e melhorias foram realizadas a partir do feedback deste segundo grupo, e assim o release da ferramenta pôde ser feito.

\section{Tecnologias Utilizadas}

A linguagem principal utilizada para escrever o código do plug-in foi o PHP, uma linguagem de servidor que é também a base para a plataforma Moodle. Ela foi utilizada em conjunto com um banco de dados MySQL e com as linguagens tradicionais de desenvolvimento Web HTML, CSS e Javascript. Também foi necessário o uso de bibliotecas complementares: o CK Editor, que é um editor de texto de código aberto; Google Maps JavaScript API e Google Charts, usadas no relatório para a exibição de localização geográfica e gráficos, respectivamente e JQuery Rate, utilizada como funcionalidade de avaliação.

A técnica de Inteligência Artificial adotada para o mecanismo de recomendação foi a filtragem baseada em conteúdo, que consiste na descrição automática do conteúdo dos itens, comparando estas características com os interesses dos usuários, para a tomada de decisão se o item é relevante ou não. Esta descrição pode ser estabelecida a partir de informações fornecidas pelo usuário ou por meio de ações realizadas por ele (REATEGUI; CAZELLA, 2006). No caso do plug-in Octopus, a decisão de recomendação é tomada ao se analisar com quais itens ou pessoas o usuário mais interage.

\section{Apresentação da Ferramenta}

O Octopus ${ }^{1}$ permite que os usuários criem e respondam postagens de diversos tópicos de discussão, de forma semelhante aos fóruns convencionais. No entanto, o plug-in também possui várias funcionalidades adicionais em comparação àqueles, as quais são ${ }^{2}$ :

- Tags: rótulos a serem aplicados para classificar as postagens por temas, favorecendo tanto a busca de postagens quanto a geração de relatórios, que permitem analisar, por exemplo, os temas mais debatidos no fórum e os autores que se destacam em determinada temática.

- Curtidas / Não curtidas: recursos que permitem que os usuários deem um feedback rápido quanto à opinião deles sobre as postagens. Como esta ideia vem das redes sociais informais, permitem uma maior familiaridade do usuário.

- Tipos de postagem: classificação de postagens de acordo com a natureza de seu conteúdo. Podem ser de três tipos: Pergunta, Narrativa e Questão Disparadora.

\footnotetext{
1 Atualmente, a ferramenta está sendo utilizada no Programa de Qualificação para Profissionais de Regulação do SUS, que pode ser acessado pelo link: http://sabertecnologias.com.br/?page_id=3536.

${ }^{2}$ Mais detalhes podem ser vistos no vídeo disponível no link: https://youtu.be/LyUKTeGVDBM.
} 
Esta última denominação é aplicada a postagens criadas apenas pelos moderadores do fórum, com o objetivo de propor discussões mais provocativas sobre os temas abordados nos cursos e incentivar a colaboração entre os participantes.

- Notificações: consiste no mecanismo de envio de informações relevantes aos usuários por e-mails. Dentre os dados informados, podem-se destacar a questão das recomendações de materiais de estudo e postagens, geradas através de algoritmo baseado nas ações realizadas pelos usuários no fórum; as postagens feitas por pessoas adicionadas à lista de contatos do usuário; curtidas e não curtidas que o usuário recebeu em suas postagens; postagens feitas pelos moderadores (questões disparadoras de discussão a fim de motivar a participação dos usuários).

- Contatos: permite que os usuários criem lista de contatos para ser notificado de suas atualizações, e enviar mensagens privadas no contexto do fórum, as quais funcionam como um chat. Sendo assim, o plug-in também incorpora a comunicação síncrona, dispensando o uso de outra ferramenta com esta finalidade.

- Pontuação: além da pontuação padrão do Moodle, o Octopus permite uma forma de pontuação extra, baseada na quantidade e natureza das interações dos usuários do fórum.

- Ranking: exibição de uma classificação geral dos usuários calculada com base em suas participações no fórum (é possível atribuir peso específico para cada um dos seguintes itens: postagens feitas, comentários feitos, curtidas recebidas), e também em outras atividades disponibilizadas no curso.

- Relatórios: é a seção onde são exibidas informações a respeito da atividade geral do fórum, que também podem ser filtradas de acordo com um usuário em particular, região geográfica, tags e data específica. Tais informações permitem que os gestores da ferramenta realizem uma análise detalhada de como as discussões estão se desenvolvendo no fórum e identifiquem, por exemplo: autores das postagens mais comentadas, mais e menos curtidas, em quantas e quais postagens determinada tag foi utilizada.

A Figura 1 demonstra o layout da tela inicial do fórum, exibindo o acesso a algumas das funcionalidades citadas anteriormente. A seção de relatórios é demonstrada na Figura 2. 
VI Congresso Brasileiro de Informática na Educação (CBIE 2017)

Anais dos Workshops do VI Congresso Brasileiro de Informática na Educação (WCBIE 2017)

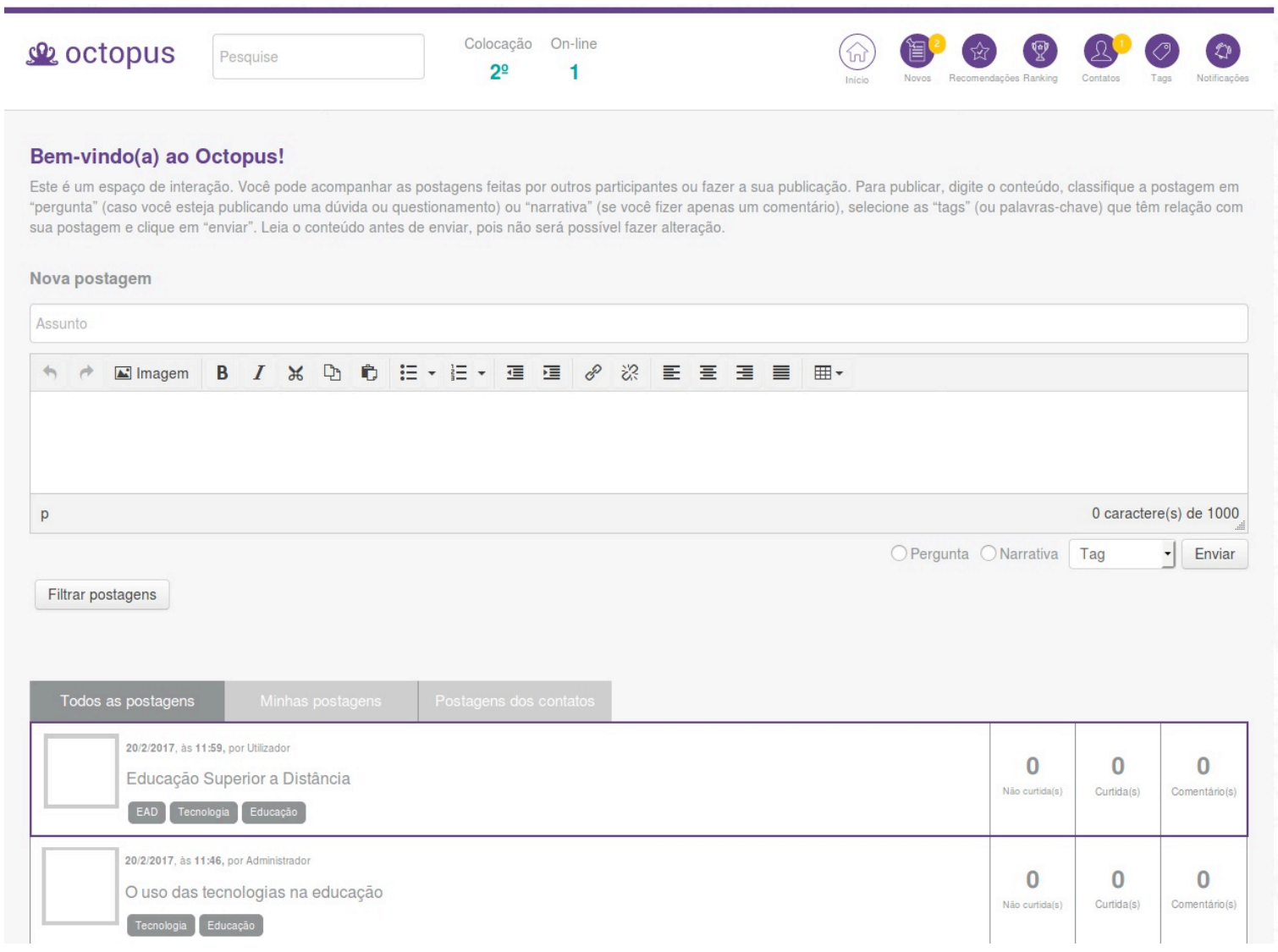

Figura 1. Tela inicial do fórum

Octopus

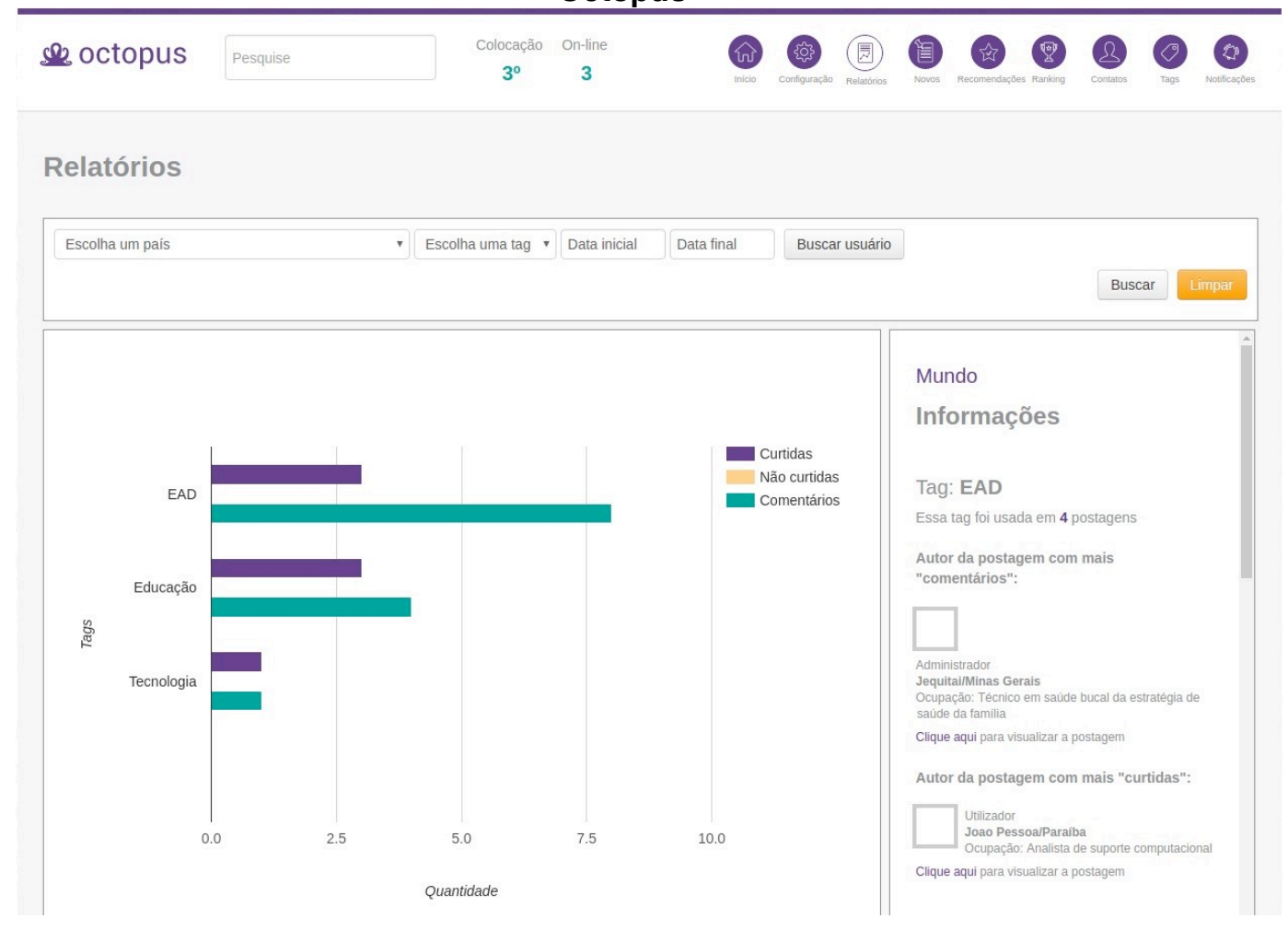

Figura 2. Seção de relatórios do plug-in 
VI Congresso Brasileiro de Informática na Educação (CBIE 2017)

Anais dos Workshops do VI Congresso Brasileiro de Informática na Educação (WCBIE 2017)

\section{Discussão de Resultados}

O plug-in, apesar de ter sua utilização relativamente recente, já tem apresentado resultados iniciais satisfatórios. Como benefícios imediatos, podemos mencionar:

- Maior eficiência no processo educativo - as funcionalidades proveem a tutores, moderadores e coordenadores recursos para atingir os seus objetivos pedagógicos, e aos alunos, uma maior satisfação em obter uma aprendizagem mais rica e eficaz. Aspectos de usabilidade incorporados também permitem que o funcionamento do sistema seja rapidamente apreendido pelos usuários, facilitando a experiência de uso.

- Maior eficiência no processo administrativo - informações obtidas por meio dos relatórios servem de entrada para o aperfeiçoamento dos cursos e indica materiais didáticos que podem ser adicionados aos cursos com a proposta de esclarecer as dúvidas mais frequentes dos participantes. O plug-in foi pensado para capturar as informações mais relevantes com essa finalidade.

Além disso, do ponto de vista institucional, a disponibilização de uma nova ferramenta implica uma importante contribuição para a comunidade de tecnologia educacional, tanto no setor de negócios como para a área acadêmica, uma vez que além do aspecto prático, o desenvolvimento deste projeto gera conhecimento científico, auxiliando na expansão do estado da arte no que concerne a objetos educacionais. Ao permitir que diversas organizações tenham acesso ao plug-in gratuitamente, podem ser obtidas mais experiências de uso que também ajudarão a embasar futuras melhorias da ferramenta, e a interação com estas instituições é uma forma de obter maior visibilidade em meio à comunidade que trabalha pela inovação na educação.

\section{Considerações Finais}

A ideia da versão inicial do Octopus foi pôr em uso um MVP (Minimum Viable product, mínimo produto viável, em português), isto é, criar um produto mais simples, com as funcionalidades principais, e receber feedback dos usuários de uma forma mais rápida e num estágio menos complexo de desenvolvimento, onde o custo de mudança é menor. No entanto, o Grupo SABER já trabalha numa versão melhorada do plug-in, baseada tanto nas informações obtidas pela sua utilização inicial quanto na realização de benchmarking de abordagens mais atualizadas, visando que esta ferramenta esteja alinhada com as mais recentes e eficazes práticas pedagógicas. Também há a previsão de lançamento de uma versão estável no diretório de plug-ins do Moodle, disponibilizando-o de forma gratuita para a comunidade.

\section{Referências}

Andresen, M. Asynchronous discussion forums: success factors, outcomes, assessments, and limitations. Educational Technology \& Society, v. 12, n. 1, p. 249-257, 2009.

Balaji, M., Chakrabarti, D. Student Interactions in Online Discussion Forum: Empirical Research from 'Media Richness Theory' Perspective. Journal of Interactive Online Learning, v. 9, n. 1, Spring 2010.

Mason, R. Student Engagement with, and Participation in, an e-Forum. Educational Technology \& Society, v. 14, n. 2, p. 258-268, 2011. 
VI Congresso Brasileiro de Informática na Educação (CBIE 2017)

Anais dos Workshops do VI Congresso Brasileiro de Informática na Educação (WCBIE 2017)

Nicholson, S. A user-centered theoretical framework for meaningful gami- fication. In: Proceedings of Games+Learning+Society 8.0. 2012, Madison, WI, p. 223-230.

Premagowrie, S., Vaani, R., Ho, R. Online Forum: A Platform that Affects Students' Learning? American International Journal of Social Science, v. 3, n. 7, December 2014.

Reategui, E., Cazella, S. Sistemas de Recomendação. In: XXV Congresso da Sociedade Brasileira de Computação. 2006, São Leopoldo, p. 306-348. 